\title{
Flow Cytomerty: Clinical Applications in Haemato-Oncology
}

\author{
Rahman $M^{1}{ }^{1}$, Giti $S^{2}$, Saha $D^{3}$
}

\begin{abstract}
In the past decade, the use of flow cytometry in the clinical haematology laboratory has grown substantially due to the development of smaller, user-friendly, less-expensive instruments and a continuous increase in the number of clinical applications. Multiple characteristics of single cells can be analyzed rapidly by flow cytometry. Both qualitative and quantitative information are obtained by flow cytometry whereas previously only in research institutions and esteemed academic centres flow cytometers were found. With advances in technology now it is possible for secondary level hospitals to use this methodology. This paper reviews the selected applications of flow cytometry in the clinical haematology laboratory in Bangladesh. This review serves to awaken the interest of stakeholders involved in the diagnosis and management of haematological malignancies $(\mathrm{HM})$ in the efficacy of flow cytometry in the immunophenotypic characterization of leukaemias and lymphomas. Relevant literature including those provided by different international consensus groups on the phenotypic characterization of HM was reviewed. Additionally, recent reports on the immunophenotypic analysis of $\mathrm{HM}$ published in haematology, oncology, pathology, immunology and cell biology journals were also analyzed. Flow cytometric immunophenotyping of $\mathrm{HM}$ is highly demanding. It is highly useful in profiling the leukaemias and lymphomas and allows proper ramification along the latest WHO classification guidelines, thereby paving the way for targeted therapy and clinical trial-driven management, significantly outweighs the cost, which can be fully recovered if properly managed. In a low-resource setting like Bangladesh, limited immunohistochemistry serves to bridge the gap in technological advancement.
\end{abstract}

Key-words: Flow cytometry, Immunophenotyping, Haematological malignancies.

\section{Introduction}

Flow cytometry is an analytic technique in which cell suspensions created from virtually any type of fresh, unfixed tissue or body fluid, including peripheral blood or bone marrow, are stained with fluorescently labelled antibodies and then subjected to analysis on a highly specialized instrument known as flow cytometer ${ }^{1}$.

Caspersson has originated the quantitative cytometry in 1930 s by the pioneering work of nucleic acid measurement of the cell ${ }^{2}$. Rapid and accurate measurements of large cell populations stimulated the development of instruments that were the forerunners of present day flow cytometers. Introduction of fluorescent dyes for measurement of total DNA content in the detection of cancer cells and fluorescent antibodies specific for cell surface markers in the separation of cell subpopulation had satisfied the need for multiparameter analysis ${ }^{1}$.

The flow cytometer consists of three main compartments: (a) sample handling: flow cell and associated fluidics; (b) light sensing: light source, optics and detectors of light scatter and fluorescence; (c) signal processing: data collection and analysis ${ }^{1}$.

Haemmatologic malignancies (HM), traditionally regarded as leukaemias, lymphomas and myelomas are in fact exceptionally heterogeneous, with WHO classification (2008) system recognizing over 60 different clinical and pathological disease subtypes. ${ }^{4}$. Over the last decade, flow cytometry has evolved from a promising new technology to an indispensable

1. Col Md Mizanur Rahman, MBBS, DCP, MCPS, FCPS, Advanced Training on Human Genetics (India), Classified Specialist in Pathology, AFIP, Dhaka; 2. Brig Gen Susane Giti, MBBS, MCPS, FCPS, Advisor Specialist in Pathology, CMH, Chitagong; 3. Maj Gen Debashish Saha, MBBS, FCPS (Chemical Pathology), MMED, Commandant and Classified Specialist in Pathology, AFIP, Dhaka. 
tool in the diagnosis of HM. Many new antibodies, improved gating strategies, and routine use of multiparameter techniques have dramatically improved the diagnostic utility of flow cytometer ${ }^{5}$.

\section{Indications of Flow Cytometric Testing:}

A group of international experts met in Bethesda, Maryland in 2006 to formulate consensus recommendations for flow cytometric testing ${ }^{6}$. The Bethesda group took a more practical approach and addressed the flow cytometric evaluation of specimens based on the clinical presentation. Consensus was reached that flow cytometric immunophenotyping is indicated in the clinical situations such as cytopenias, especially bicytopenia and pancytopenia, elevated leucocyte count, including lymphocytosis, monocytosis and eosinophilia, presence of atypical cells or blasts in the peripheral blood, bone marrow or body fluids, plasmacytosis or monoclonal gammopathy, organomegaly and tissue masses. In these clinical situations, flow cytometric immunophenotyping can provide a sensitive screen for the presence of haematologic malignancy and assist in demonstrating the absence of disease ${ }^{7}$.

Besides these, flow cytometry may be used as an important tool for staging a previously diagnosed haemato-lymphoid neoplasm, monitoring response to treatment including detection of minimal residual disease (MRD), documenting relapse or progression, and diagnosing an intercurrent haemmatologic malignancy, such as a therapy-related myelodysplastic syndrome (MDS) ${ }^{7}$.

\section{Information Provided by Flow Cytometry Immuno-} phenotyping of Haematologic Malignancies:

After. receiving a specimen for flow cytometric testing, a decision is to be made to evaluate the cell lineages and antigens basing on the type of specimen and other available information, such as medical indication of testing listed in the requisition, clinical history, morphologic findings, history of prior flow cytometric testing, results of other laboratory testing and possibly results of any preliminary screening testing performed in the flow cytometric laboratory. Following this approach, a rapid screen for haematologic neoplasms can be assessed by flow cytometric immunophenotyping of clinical specimens and thus play a key role in diagnosis and classification $^{8}$.
In the assessment for haematologic malignancies, several steps are taken in the application and interpretation of this immunophenotypic information: Identification of cells from different lineages and determination of whether they are mature or immature, such as detection of mature B-lymphoid cells and myeloblasts, detection of abnormal cells through identification of antigen expression that differs significantly from normal, detailed documentation of the phenotype of abnormal cell populations, documentation of increased or decreased intensity of staining by fluorochrome labeled antibodies, evaluation of whether the information available is diagnostic of a distinct disease entity and, if not, development of a list of possible entities with suggestion of additional studies that might be of diagnostic value such as immunohistochemistry, conventional cytogenetic, Fluorescence In Situ Hybridization (FISH) and molecular diagnostic studies and lastly provision of immunophenotypic information that might be of additional prognostic value, including the identification of targets for potential directed therapy ${ }^{8}$.

\section{Identification and Enumeration of Blasts:}

Blasts often differ from more mature cells by expressing markers of immaturity and lacking antigens expressed by more mature cells. For example, myeloblasts can be distinguished from maturing myeloid cells if they display low orthogonal (side) light scatter, markers of immaturity such as CD34 and CD117, and lack markers of maturation such as CD11b, CD15 and CD16. Immature B-lymphoid cells can be distinguished from mature B-lymphoid cells if they express CD34 and Terminal deoxynucleotidyl transferase (TdT), and lack surface immunoglobulin and CD20. Immature T-lymphoid cells can be distinguished from mature T-lymphoid cells if they express CD34 and TdT, or CD1a or lack surface expression of CD3. A plot of CD45 versus orthogonal (side) light scatter is very useful in identifying blasts by their low side light scatter and weak intensity expression of CD45. This representation can help to distinguish blasts from lymphocytes (bright CD45), erythroid precursors (essentially negative CD45), neutrophilic precursors and eosinophils (higher side light scatter and brighter CD45). However, basophils usually fall within the blast region in this plot due to loss of granules during processing. Through the analysis of 
many thousands of cells, flow cytometric studies can determine the precise percentage of the total cells analyzed that demonstrate the phenotypic features of immature cells ${ }^{8}$.

\section{Assignment of Blast Lineage:}

For the selection of appropriate therapy, reliable distinction between AML and ALL is essential. AML usually express antigens characteristic of neutrophilic or monocytic differentiation such as CD13, CD15, CD33, CD64, CD117, and myeloperoxidase. In ALL, CD19 has the highest sensitivity and specificity for the detection of B-cell lineage and cytoplasmic CD3 for the detection of T-cell lineage. Cytoplasmic CD22 is also a sensitive and specific B-lineage marker. Leukaemic blasts may aberrantly express antigens from another lineage. Lymphoid antigens that are frequently expressed in AML include CD7, CD56, CD2 and CD19. ALL frequently demonstrates expression of one or more myeloid antigen. Although the detection of 1 or 2 myeloid antigens on lymphoblasts can assist in the identification of the abnormal cells, it does not appear to be of independent prognostic feature in ALL, and should not be used to diagnose biphenotypic leukaemia ${ }^{8}$. If the leukaemic blasts express antigens from more than one cell line or demonstrate very few lineage-associated antigens, it would be difficult to determine the lineage. With the advent of detailed flow cytometric immunophenotyping, approximately $5 \%$ of acute leukaemias were found to demonstrate lineage heterogeneity, either biphenotypic leukaemia (expression of antigens from more than one lineage by single population of blasts) or bilinear leukaemia (2 populations of blasts from different lineages) ${ }^{9}$.

\section{Acute Leukaemia:}

Flow cytometric analysis of acute leukaemia is interpretive, combining the pattern and intensity of antigen expression to reach a definitive diagnosis ${ }^{7}$. Gating is critical to isolate the abnormal cells because the leukaemic phenotype should be determined on as pure a population as possible. Most leukaemias involve the analysis of bone marrow. Standard forward and side scatter gating is not optimal for separating bone marrow cells because of the overlap between monocytes, blasts, myelocytes, promyelocytes and metamyelocytes. As bone marrow cells mature, they express increasing CD45. Thus, when CD45 is combined with side scatter, which separates lineages based on cytoplasmic complexity, the bone marrow sample is readily separated into cellular constituents. Infiltration of marrow by mature cells or blasts is more easily recognized on a CD45 versus side-scatter plot than on traditional forward side-scatter gating ${ }^{10}$.

Advances in flow cytometry hardware and software and the development of new dyes have resulted in the routine use of multicolour and multiparametric flow cytometry. This approach has advantages over double and triple colour measurements in the detection of minimal residual disease in $A M L$ and in ALL (e.g., using CD45/CD19/CD10 and TdT), the enumeration of the proportion of clonal plasma cells in multiple myeloma, and the detection in the leukaemic cells of certain molecules that are widely expressed in normal blood cells (e.g., ZAP-70 in chronic lymphocytic leukaemia) ${ }^{11}$.

\section{Immunological Markers in Acute Leukaemia:}

Though the major role of flow cytometry is to provide immunophenotypic data, cellular morphology can be examined both by forward-side scatter and CD45side scatter analysis ${ }^{12}$. The ability of flow cytometry to identify myeloid versus lymphoid differentiation has approached $98 \%$. Immuno- phenotyping plays a central role in the determination of clinically relevant subsets $^{13}$. Although there are a large number of Monoclonal antibody (McAb-recognizing antigens) of haemopoietic cells, for practical reasons a well-defined set of reagents need to be selected for the study of cases of acute leukaemia. An initial McAb panel should help to distinguish AML from ALL and to further classify ALL into B- or T-cell lineage. Panel of monoclonal antibodies for the diagnosis of acute leukaemias are shown in Table-l. This panel is constituted as follows: B-lymphoid markers: CD19, CD10 and cytoplasmic CD22 and CD79a, T-lymphoid markers: CD2, CD7 and cytoplasmic CD3, Myeloid markers: CD13, CD33, CD117 and cytoplasmic myeloperoxidase (anti- MPO) and non lineage specific markers: CD34, HLA -DR and $\mathrm{TdT}^{14}$.

Two aspects that need to be considered are the degree of lineage specificity of the antigen and whether it is expressed in the membrane or the cytoplasm. Some markers are highly specific and 
sensitive for a particular lineage (e.g., cCD3 for T cells, CD79a for B-cells and anti-MPO for myeloid cells), whereas others (e.g., CD10, CD13, or CD7) are less lineage specific. The second aspect that take into account when performing immunophenotyping is that the most specific markers are either expressed earlier in the membrane during cell differentiation (e.g., CD3) or they are only detectable in the cytoplasm (e.g., anti-MPO, CD79a) or both $^{15}$.

A second set of McAb is necessary to identify ALL further into the various subtypes and to identify rare cases of AML derived from cells committed to the megakaryocytic and erythroid lineages. This set comprises cytoplasmic and membrane Ig staining in B-lineage ALL; CD1a, CD4, CD5, CD8 and anti-TCR in T-lineage ALL; and, in $A M L$, antibodies that detect membrane glycoproteins present in platelets and megakaryocytes or glycophorin A expressed by erythroid precursors ${ }^{16}$.

Role of flow cytometric immunophenotyping in the diagnosis and classification of AML:

Flow cytometric immunophenotypic studies remain of value in its distinction from ALL. In addition, flow cytometric studies are also of value in the identification of megakaryocytic differentiation with expression of CD41, CD61 and pure erythroid leukaemia with expression of CD235a (glycophorin A) or CD36 in the absence of CD64, myeloperoxidase, and other myeloid-associated antigens. Although flow cytometric studies can also evaluate for monocytic differentiation, cytochemical stains remain part of the current WHO classification scheme. Flow cytometric evaluation of CD14 lacks sensitivity for the detection of monocytic differentiation ${ }^{17}$. However, it has been suggested that the sensitivity of the flow cytometric assay can be improved by evaluation of other monocyteassociated antigens such as co-expression of CD36 and CD64 bright, intermediate CD15 plus bright CD33, and different antibodies directed against different CD14 epitopes ${ }^{18}$.

Some phenotypes in AML are associated with the presence of recurrent genotypic abnormalities. For example, AML with $t(8 ; 21)$ is associated with aberrant expression of CD19, CD56 and sometimes TdT. Acute promyelocytic leukaemia with $t(15 ; 17)$ often has the following phenotype: CD34- or only partially positive, HLA-DR- or only partially positive, CD11b, CD13 heterogeneous, CD117, CD33 (homogenous bright positive), and CD15 or weak intensity staining. Recently a similar CD34-, HLA-DR- phenotype has been described in a subset of AML with cup-shaped nuclear invaginations, normal cytogenetics, and an apparent association with FLT-3 gene with internal tandem duplication ${ }^{19}$. Therefore, although flow cytometric immunophenotypic studies may be used as a screening tool, they lack specificity and sensitivity for the detection of genotypic abnormalities ${ }^{20}$.

Role of flow cytometric immunophenotyping in the diagnosis and classification of ALL:

Identification of B-cell or T-cell lineage and assessing response to treatment, including the identification of early responders and the detection of minimal residual disease (MRD) ${ }^{21}$. Table-II and III shows the pattern of markers for the diagnosis of B-ALL and T-ALL.

\section{Immunophenotyping in Chronic Lymphoproli- ferative Disorders:}

Flow cytometric immunophenotypic study is essential for the diagnosis and characterization of the lymphoproliferative disorders. Immunological markers help to distinguish lymphoblastic leukaemias and lymphoblastic lymphomas, which are $\mathrm{TdT}(+)$ positive, from mature or chronic lymphoid diseases, which are consistently TdT (-) negative. Immunophenotyping also enables whether the malignant cells are of $B$ - or $T$ - lymphoid nature and demonstrates clonality in the $B-$ cell cases ${ }^{11}$.

\section{Panel of McAb for diagnosis and classification of} chronic lymphoproliferative disorders:

The diagnosis of a B - or $\mathrm{T}-$ cell disorder requires a small battery of McAb. It is convenient to use two-step procedure with an initial panel applicable to all cases and a second panel based on the results of the first panel and the tentative diagnosis by clinical features and/or cell morphology ${ }^{22}$.

The first panel of markers is intended to distinguish B-cell from T-cell disorders, to demonstrate B-cell clonality, to confirm the diagnosis of Chronic Lymphocytic Leukaemia (CLL) and to confirm or exclude a non-CLL B - cell neoplasm. Panel of monoclonal antibodies for the diagnosis of lymphoid disorders are shown in Table-IV. 
Table-I: Panel of monoclonal antibodies for the diagnosis of acute leukaemias ${ }^{24}$.

\begin{tabular}{|c|c|c|c|}
\hline \multirow{2}{*}{$\begin{array}{c}\text { Type of } \\
\text { panel }\end{array}$} & \multicolumn{2}{|l|}{ ALL } & \multirow[t]{2}{*}{ AML } \\
\hline & B - lineage & $\mathrm{T}$ - lineage & \\
\hline First - line & CD19, CD22, CD79a, CD10 & CD7, CD2, cCD3 & $\begin{array}{c}\text { CD13, CD33, CD117, } \\
\text { anti - MP0 }\end{array}$ \\
\hline \multicolumn{4}{|c|}{ TdT, HLA -DR, CD34 } \\
\hline Second - line & су $\mu$, SmIg & $\begin{array}{c}\text { CD1a, CD5, CD4, } \\
\text { CD8, anti-TCR }\end{array}$ & $\begin{array}{l}\text { CD41, CD42, CD61, anti- } \\
\text { glycophorin A }\end{array}$ \\
\hline
\end{tabular}

ALL=Acute Lymphoblastic Leukaemia; $\mathrm{AML}=$ Acute Myeloblastic Leukaemia; $\mathrm{CD}=$ Cluster differentiation; $\mathrm{TdT}=$ Terminal deoxynucleotidyl transferase; Smlg=Surface membrane immunoglobulin; TCR=T- cell receptor.

CD10 and cy $\mu$ are not essential for a diagnosis of B - lineage ALL but they are important in paediatric cases to identify common-ALL, pro-B-ALL and pre-B-ALL.

Table-II: Diagnosis of acute leukaemia (B-ALL) ${ }^{5}$.

\begin{tabular}{|c|c|c|c|c|}
\hline Markers & $\begin{array}{c}\text { Precursor B- } \\
\text { ALL }\end{array}$ & Common ALL & Pre-B-ALL & Mature B -ALL \\
\hline HLA - DR & \multirow{4}{*}{\multicolumn{4}{|c|}{ Positive }} \\
\hline cCD22 & & & & \\
\hline CD79a & & & & \\
\hline CD19 & & & & \\
\hline $\mathrm{TdT}$ & \multicolumn{3}{|c|}{ Positive } & Negative \\
\hline CD10 & Negative & \multicolumn{2}{|c|}{ Positive } & Negative \\
\hline сун & \multicolumn{2}{|c|}{ Negative } & Positive & Negative \\
\hline sIg & \multicolumn{3}{|c|}{ Negative } & Positive \\
\hline
\end{tabular}

Table-III: Diagnosis of acute leukaemia (T-ALL) ${ }^{5}$.

\begin{tabular}{|c|c|c|c|c|}
\hline Markers & Pro T- ALL & Pre-T-ALL & $\begin{array}{c}\text { Cortical-T- } \\
\text { ALL }\end{array}$ & Mature T-ALL \\
\hline $\mathrm{TdT}$ & \multicolumn{3}{|c|}{ Positive } & Negative \\
\hline cCD3 & \multicolumn{4}{|c|}{ Positive } \\
\hline CD7 & \multicolumn{4}{|c|}{ Positive } \\
\hline CD2 & Negative & \multicolumn{3}{|c|}{ Positive } \\
\hline CD5 & Negative & \multicolumn{3}{|c|}{ Positive } \\
\hline CD4 & \multicolumn{2}{|c|}{ Negative } & Positive for & Positive for CD4 \\
\hline CD8 & \multicolumn{2}{|c|}{ Negative } & CD4 and CD8 & or CD8 \\
\hline CD1a & \multicolumn{2}{|c|}{ Negative } & Positive & Negative \\
\hline sCD3 & \multicolumn{2}{|c|}{ Negative } & & Positive \\
\hline
\end{tabular}

Table-IV: Panel of monoclonal antibodies for the diagnosis of lymphoid disorders ${ }^{24}$.

\begin{tabular}{|c|c|c|}
\hline Type of panel & B cell & T cell \\
\hline First -line & SmIg (kappa/lambda) & CD2, CD5* \\
& CD19, CD23, FMC7 & \\
\hline SmCD79b, smCD22, CD5* & CD3, CD4 \\
& CD11c, CD25, CD103 & CD7, CD8 \\
\hline
\end{tabular}


*B-cell subset and T-cell marker. Optional markers: CD25, CD79a and natural killer associated (e.g. CD16, CD56, CD57, and CD11b). Cylg: cytoplasmic immunoglobulin. Smlg: surface membrane immunoglobulin.

Table-V: Scoring system for the diagnosis of chronic lymphocytic leukaemia ${ }^{24}$.

\begin{tabular}{|l|c|c|}
\hline \multicolumn{1}{|c|}{ Marker } & Points & Points \\
\hline & 1 & 0 \\
\hline CD5 & Positive & Negative \\
\hline CD23 & Positive & Negative \\
\hline FMC7 & Negative & Positive \\
\hline SmIg & Weak & Moderate/Strong \\
\hline CD22/CD79b & Weak/Negative & Moderate/Strong \\
\hline
\end{tabular}

${ }^{*}$ Scores for CLL range from 3 to 5 , whereas in the other B-cell disorders they range from 0-2.

The results obtained with the first - line panel of McAbs can be combined with scoring system to establish the diagnosis of CLL and to distinguish CLL cases with atypical morphology and CLL with increased numbers of prolymphocytes (CLL/PL) from other B-cell diseases such as B-cell prolymphocytic leukaemia (B-PLL) and B-cell lymphoma in leukaemic phase ${ }^{22}$. Scoring system for the diagnosis of chronic lymphocytic leukaemia is shown in Table-V.

When the marker profile of using the first-line panel of McAb yields a B-cell phenotype not typical of $C L L$, a second panel of McAb can be used. This is selected in the light of the review of the morphology, clinical information, or other laboratory features. Expression of other B-cell markers such as CD11c, CD25, CD103 and HC2 may need to be investigated to distinguish hairy cell leukaemia $(\mathrm{HCL})$ from other disorders with circulating villus cells that may be confused with $\mathrm{HCL}$, such as splenic lymphoma with villous lymphocytes (SLVL) or marginal zone lymphoma and the HCL variant ${ }^{23}$. When the first-line panel of markers suggest a T-cell phenotype $(\mathrm{CD} 2+, \mathrm{CD} 5+/-)$, expression of other T-cell markers such as CD3, CD7, CD4 and CD8 may need to be investigated. CD25 may be used in cases of suspected adult $T$ - cell leukaemia lymphoma. When markers do not indicate either $B$ lineage or $T$ lineage, testing for NK cell markers should be considered.

\section{Conclusion}

The clinical application of flow cytometric immunophenotypic study for the diagnosis of different diseases is vast and widespread and its detailed discussion is beyond the scope of the present review article. Flow cytometric immuno- phenotypic study of haematological malignancies has become absolutely essential in view of its role in clearly defining the complex haemopoietic malignancies according to lineage and maturation stage, using differentiation markers. It is also fast, specific and sensitive. Flow cytometer is now available in a number of centres in the capital city of Bangladesh and though it appears expensive to maintain, but careful planning, including having a regional centre in the divisional headquarters of Bangladesh to start with, along with meticulous management coupled with its wide benefit will offset the cost.

\section{References}

1. Paraskevas F. Clinical Flow Cytometry. In: Lee GR, Foerster J, Lukens J, Paraskevas F, Greer JP, Rodgers GM, editors. Wintrobe's Clinical Haemmatology. Baltomore: Williams and Wilkins; 1993. p. 56-70.

2. Caspersson TO, Schult J. Nucleic acid metabolism of the chromosomes in relation to gene reproduction. Nature 1938; 142: 294.

3. Coons $\mathrm{AH}$, Kaplan $\mathrm{MH}$. Localization of antigen in tissue cells. Improvements in a method for the detection of antigen by means of fluorescent antibody. J Exp Med 1950; 91: 1.

4. Kamentsky LA, Melamed MR, Derman H. Spectrophotometer: New instrument for ultrarapid cell analysis. Science 1965; 150: 630-1.

5. Olaniyi JA. Flow cytometric immunophenotyping of haematological malignancies: the way forward in Nigeria. Pathology and Laboratory Medicine International 2011; 3: 17-24. 
6. Orfao A, Lopez A, Flores $J$ et al. Diagnosis of haematological malignancies: new applications for flow cytometry. The European Haemmatology Association Education Program, 2006; 2(1): 6-12.

7. Jennings CD, Foon KA. Recent Advances in Flow Cytometry: Application to the Diagnosis of Haematologic Malignancy. Blood 1997; 90 (8):2863 -52 .

8. Wood B. 9-color and 10-color flow cytometry in the clinical laboratory. Arch Pathol Lab Med 2006; 130: 680-90.

9. Jaffe ES, Harries NL, Stein H. Tumours of haemopoietic and Lymphoid Tissues. World Health Organization Classification of Tumours Lyon, France: IARC Press; 2001.

10. Stetier-Stevenson M, Davis B, Wood B et al. 2006 Bethesda International Consensus Conference on Flow Cytometry Immunophenotyping on Haematolymphoid Neoplasia. Cytometry B Clin Cytom 2007; 72B:S3.

11. Davis BH, Holden JT, Bene MC et al. 2006 Bethesda International Consensus recommendations on the flow cytometric immunophenotypic analysis of haematolymphoid neoplasia: medical indications. Cytometry B Clin Cytom 2007; 72B: S5 -S13.

12. Borowitz MJ, Guenther KL, Shults KE et al. Immunophenotyping of acute leukaemia by flow cytometric analysis. Use of CD45 and right-angle light scatter to gate on leukaemic blasts in three-color analysis. Am J Clin Pathol 1993;100:534.

13. Bernier $\mathrm{M}$, Massey $\mathrm{M}$, Deleuw $\mathrm{N}$ et al. Immunological definition of acute minimally differentiated myeloid leukaemia (MO) and acute undifferentiated leukaemias (AUL). Leuk Lymphoma 1995; 18:13.

14. Ueken FM, Sather $H$, Rahman $G$ et al. Leukaemic cell growth in SCID mice as a predictor of relapse in high-risk B-lineage acute lymphoblastic leukaemia. Blood 1995; 85: 873.

15. Buccheri V, Shetty V, Yoshida N. The role of an anti-myeloperoxidase antibody in the diagnosis and classification of acute leukaemia: a comparison with light and electron microscopy cytochemistry. British Journal of Haemmatology, 1992; 80: 62-8.
16. McKenna RW, Washington LT et al. Immunophenotypic analysis of haematogones (B lymphocyte precursors) in 662 consecutive bone marrow specimens by 4-color flow cytometry. Blood 2001; 98: 2408-507.

17. Brunning RD, Matutes E, Borowitz M. Acute leukaemia of ambiguous lineage. In: Jafe ES, Harris NL, Stein H, Vardiman JW, editors. World Health Organization classification of Tumours, 2001; Tumours of haemopoietic and lymphoid tissues, $p$. 27. IARC press, Lyon.

18. Dunphy $\mathrm{CH}$, Orton SO, Mantell J. Relative contributions of enzyme cytochemistry and flow cytometric immunophenotyping to the evaluation of acute myeloid leukaemias with a monocytic component and of flow cytometric immunophenotyping to the evaluation of absolute monocytoses. Am J Clin Pathol 2004; 122: 865-74.

19. Krasinskas AM, Wasik MA, Kamoun $M$ et al. The usefulness of CD64, other monocyte-associated antigens and CD45 gating in the subclassification of acute myeloid leukaemias with monocytic differentiation. Am J Clin Pathol 1998; 110: 797-805.

20. Kussick SJ, Stirewelt GL, Yi HS. A distinctive morphology in acute myeloid leukaemia is strongly associated with loss of HLA-DR expression and FLT-3 internal tandem duplication. Leukaemia 2004; 18: 1591-6.

21. Arber DA. Realistic pathologic classification of acute myeloid leukaemia. Am J Clin Pathol 2001; 115: 552-60.

22. Matutes E, Owusu-Ankomah K, Morilla R. The immunological profile of $B$ - cell disorders and proposal of a scoring system 1994; 8: 1640-5.

23. Matutes E, Morilla R, Owusu-Ankomah K. The immunophenotype of hairy cell leukaemia $(\mathrm{HCL})$ : proposal for a scoring system to distinguish BCL from B-cell disorders with hairy or B-cell disorders with hairy or villous lymphocytes. Leukaemia and lymphoma 1994; 14 (suppl 1): 57-61.

24. Matutes E, Morilla R, Morilla AM. Immunophenotyping. In Bain BJ, Bates I, Laffan MA, Lewis SM, editors. Dacie and Lewis Practical haematology. Churchill Livingstone; 2011. p.353-71. 